\title{
O IMPACTO DA VOLATILIDADE CAMBIAL NO COMÉRCIO ENTRE OS PAÍSES DO NAFTA
}

\section{THE IMPACT OF THE REAL EXCHANGE RATE VOLATILITY ON TRADE BETWEEN NAFTA COUNTRIES}

\author{
Rodrigo Henrique Bosco* \\ Maurício Vaz Lobo Bittencourt ${ }^{* *}$
}

\begin{abstract}
RESUMO
Dentre as questões relevantes em comércio internacional, tem-se: Qual a importância do comércio internacional para o crescimento econômico de um país? Qual o impacto da volatilidade cambial no comércio entre países? Na tentativa de responder tais questões, este artigo se propôs a estimar o impacto da volatilidade da taxa de câmbio real no comércio internacional dos países do NAFTA (período 2001-2014), através da estimação de um modelo gravitacional considerando três medidas de volatilidade cambial aplicados a oito diferentes setores, e por fim, para o comércio total. Para o total de comércio, somente a medida de volatilidade IGARCH foi estatisticamente significativa e o coeficiente estimado apresentou sinal negativo como esperado teoricamente, sendo assim, a volatilidade cambial é prejudicial para o comércio dos países integrantes do Nafta. Agricultura, manufaturas, maquinaria e transportes e têxteis apresentaram coeficientes de volatilidade negativos e estatisticamente significativos em pelo menos umas das medidas de volatilidade.
\end{abstract}

Palavras-chave: Comércio, NAFTA, volatilidade cambial, modelo gravitacional.

\begin{abstract}
Among the relevant questions in international trade, we have: What is the importance of international trade for the economic growth of a country? What is the impact of exchange rate volatility on trade between countries? In an attempt to answer such questions, this paper aims to estimate the impact of real exchange rate volatility on the international trade of NAFTA countries (period 2001-2014) by estimating a gravity trade model considering three measures of exchange rate volatility applied to eight different sectors, and finally for total trade. For the total trade, only the IGARCH volatility measure was statistically significant and the estimated coefficient presented negative sign as theoretically expected, thus, the exchange rate volatility is detrimental to the trade of NAFTA member countries. Agriculture, manufacturing, machinery and transport and textiles showed negative and statistically significant volatility coefficients in at least one of the volatility measures.
\end{abstract}

Keywords: Trade, NAFTA, exchange rate volatility, gravity model.

Graduado em Economia pela Universidade Federal do Paraná

"Doutor em Desenvolvimento Econômico e Comércio Internacional pela The Ohio State University 


\section{INTRODUÇÃO}

Com o comércio, além da produção em escala que gera produto e renda, há uma diversificação da oferta de produtos disponíveis aos consumidores dos países parceiros, aumentando dessa forma seu bem-estar. A especialização de cada país no produto que possui vantagem na produção, permite a produção em escala deste produto (Jesus, 2010), porém, nota-se que alguns países procuram produzir uma gama muito ampla de produtos diferentes, os quais nem sempre possui vantagem na produção, indo no sentido contrário ao que se refere as vantagens comparativas. Pode-se entender que a especialização não é a única variável a influenciar o comércio internacional, pois outras variáveis como PIB, população, preferências dos consumidores, distância, custos de transação, tarifas e câmbio podem influenciar positivamente ou negativamente o comércio.

As relações comerciais e proximidades geográficas entre Estados Unidos, Canadá e México facilitam o fluxo de comércio e investimentos entre esses países, favorecendo as negociações para a criação de um bloco de integração regional. É nesse cenário de integração regional que os interesses dos grupos privados cedem espaço aos interesses dos mercados integrados e as estratégias de competitividade regionais. A formação de blocos regionais favorece os países membros no que concerne as estratégias nacionais de competição e alavancagem de capacidade competitiva, ampliando a geração de riquezas e utilizando as economias de escala para redução dos custos de transporte (Ministério das Relações Exteriores/FUNCEX, 1993).

Em agosto de 1992, Estados Unidos, Canadá e México finalizaram as negociações do Acordo de Livre Comércio da América no Norte (North American Free Trade Agreement) conhecida como NAFTA. Os integrantes de uma Zona de Livre Comércio, como é o caso dos países do NAFTA, se prontificam a eliminar progressivamente ao longo do tempo barreiras e obstáculos sobre a negociação de produtos entre eles (Ratti, 1997). Cada país possui liberdade em relação a qual política interna, e quais tarifas impor sobre produtos de terceiros, diferente de outros blocos regionais como o Mercosul e a União Europeia (Ratti, 1997).

Inicialmente, a criação de blocos de integração regional como o NAFTA trazem benefícios como eliminação de barreiras ao comércio e aos investimentos, economias de escala e vantagens competitivas comerciais (Ministério das Relações Exteriores/FUNCEX, 1993). Existem inúmeras questões relevantes no que se refere à criação de blocos de integração regional no que concerne o comércio internacional. Pode-se citar duas: Qual a importância do comércio internacional para o crescimento econômico de um país? Qual o impacto da volatilidade cambial no comércio entre países? Na tentativa de responder tais questões, este artigo se propõe a estimar o impacto da volatilidade da taxa de câmbio real no comércio internacional dos países do NAFTA e demonstrar a importância das políticas monetárias para o comércio e para o crescimento econômico desses países. Será estimado o impacto da volatilidade do câmbio no comércio entre os países do NAFTA, para os seguintes setores: agricultura, têxtil, metais e minérios, combustíveis, alimentos, maquinaria e transportes, químico, manufaturas e para o total do comércio. Adicionalmente ao impacto da volatilidade cambial sobre o comércio dentro do bloco regional, variáveis como o $\mathrm{PIB}$, distância e a volatilidade de um terceiro país serão incluídas no modelo.

Este artigo se divide em seis seções, incluindo esta introdução. A próxima seção se encarrega da revisão de literatura de trabalhos semelhantes, em seguida tem-se uma discussão sobre a metodologia a ser utilizada, resultados e discussões acerca da pesquisa e, por fim, as considerações finais.

\section{REVISÃO DE LITERATURA}

O estudo acerca das relações comerciais entre os países pode ser aprimorado através do uso dos modelos gravitacionais para estimar o impacto e a significância de algumas variáveis no comércio internacional. Carmo e Bittencourt (2013) em seu trabalho sobre o impacto da volatilidade cambial no comércio internacional, refere-se ao modelo gravitacional como uma solução em forma de equação reduzida de um sistema de equilíbrio geral de comércio, onde o comércio internacional depende de variáveis como tamanho do país, distância entre os parceiros comerciais, estágio de desenvolvimento e grau de abertura dos mercados.

Para o modelo de equilíbrio Clark et al. (2004) ressalta a importância das variáveis macroeconômicas para melhor entender a relação entre volatilidade cambial e comércio. Para os autores, a única variável do modelo que muda é a volatilidade cambial, que 
pode ter diferentes formas de mensuração e, quanto a isso, Willett (1986) chama a atenção para o cuidado na mensuração da volatilidade, pois dependendo das estimativas estas podem levar a resultados equivocados.

Anderson e Van Wincoop (2004) mostram que o modelo gravitacional pode ser estimado de forma não linear por Mínimos Quadrados Ordinários através do modelo de efeitos fixos, que permitem a inclusão de termos de resistência multilateral, de modo a evitar viés causado pela omissão de variáveis que estariam contidas no termo de erro da regressão (Mendonça, 2011).

Cheng e Wall (2005) estimaram o modelo gravitacional utilizando uma base de dados de 3.188 observações resultado da relação unidirecional de 797 pares de países, sendo os países integrantes, países industrializados ou membros da OCDE. Utilizando a modelagem de corte transversal agrupada (pooled-cross-section) e o modelo de efeitos fixos com e sem restrições, os autores observaram que o modelo de corte transversal agrupado resulta em estimativas viesadas para os parâmetros do modelo através do problema de variáveis omitidas. Quanto ao modelo de efeitos fixos, foram testados três tipos de restrições sobre os parâmetros, sendo uma delas a diferenciação das variáveis. Foi demonstrado que as formas alternativas para estimação do modelo de efeitos fixos impondo restrições nos parâmetros não possuem suporte estatístico, e também não possuem fundamento econômico para serem mantidas, portanto, o modelo de efeitos fixos pode ser estimado sem a imposição de restrições resultando em estimativas não enviesadas dos parâmetros.

Conclusões de estudos a respeito do impacto da volatilidade da taxa de câmbio sobre o comércio internacional são bastante ambíguas. Utilizando um modelo gravitacional estimado pela técnica de dados em painel para o Mercosul, Bittencourt, Larson e Thompson (2007) encontrou coeficientes estatisticamente significativos e negativos para a volatilidade da taxa real de câmbio. Nesse estudo, a volatilidade do câmbio se mostrou prejudicial para o comércio setorial do Mercosul no período analisado de 1989 a 2002.

Campos e Bittencourt (2014) estimou, através da modelagem em painel, o impacto da instabilidade da taxa real de câmbio de médio e longo prazo para os setores Agropecuário, Máquinas e Equipamentos de Transportes, Químico, Metais e Minerais e
Manufaturados, encontrando significância estatística para os coeficientes que representam a volatilidade da taxa real de câmbio e impactam negativamente o comércio entre o Brasil e seus principais parceiros comerciais no período de 1989 a 2011.

Utilizando o quadrado dos resíduos de um modelo ARIMA para a taxa real de câmbio, através da modelagem de correção de erros Asseery e Peel (1991) observaram que a volatilidade da taxa real de câmbio possui influência positiva e significativa no comércio entre Austrália, Japão, Estados Unidos e Alemanha Ocidental para o período de 1972 a 1987. A respeito do impacto positivo da volatilidade cambial sobre o comércio, Franke (1991) mostra que este é factível, visto que as firmas reajustam as quantidades exportadas de forma a sempre otimizar o fluxo de caixa esperado. De acordo com o autor, incrementos na volatilidade cambial podem ser oportunidades de lucro frente aos custos de entrada e saída associados a atividade comercial, possibilitando ganhos semelhantes àqueles obtidos com opções de ações, dessa forma, o comércio irá acontecer sempre que houver oportunidades de lucro.

Também utilizando a modelagem de séries temporais para a volatilidade cambial, Toda (2012) estimou um modelo gravitacional para as exportações do Brasil com relação aos seus principais parceiros comerciais, utilizando como medida de volatilidade a modelagem de séries temporais EGARCH (Exponential GARCH), Peree e Steinherr e desvio padrão móvel para o período de 1999 a 2010. Em seu trabalho encontrou significância estatística para as três medidas de volatilidade em um modelo que tinha como variável explicada o total do comércio entre o Brasil e seus parceiros comerciais.

A literatura a respeito do impacto da volatilidade do câmbio no comércio é muito ampla e os resultados encontrados em cada trabalho podem ser muito distintos. Com um modelo de correção de erros, Bahmani-Oskooee e Scott (2009) usou dados de exportações e importações entre México e Estados Unidos (EUA) para o período de 1962 a 2004, incluindo 102 produtos industriais, para estimar o impacto da volatilidade da taxa real de câmbio e da renda no comércio bilateral, concluindo que a maior parte dos produtos industriais são afetados negativamente no curto prazo, tendo como exemplo produtos agrícolas e têxteis. 
Para o total do comércio americano (exportações e importações), Augustine (1995) verifica que a volatilidade da taxa real de câmbio não exerce nenhum impacto, no entanto, para o longo prazo o modelo de correção de erros aponta que para as exportações, 32 dos 102 produtos industriais sofrem efeitos da volatilidade cambial, sendo que dos 32, 21 produtos sofrem impactos negativos e 11 impactos positivos. No curto prazo para o modelo de correção de erros, 61 produtos industriais são influenciados pela volatilidade cambial, alguns positivamente e outros negativamente. Por fim, a variável dummy acrescentada no modelo representando o NAFTA é significativa, mostrando que a criação do bloco teve influência no comércio bilateral entre México e EUA.

Augustine (1995) utilizando a modelagem de cointegração e correção de erros concluiu que a volatilidade da taxa real de câmbio impacta negativamente o total das exportações americanas para o período de 1973 a 1991. Mensurando três medidas de volatilidade, sendo elas médias móveis, modelos ARCH e modelo de momento linear, o autor concluiu que a volatilidade cambial além de afetar o comércio, pode impactar significativamente na alocação de recursos.

Chowdhury (1993) estimou um modelo de correção de erros para avaliar o impacto da volatilidade da taxa real de câmbio no comércio entre os países do G-7, assumindo que os agentes são avessos ao risco o autor concluiu que a volatilidade cambial reduz o comércio, altera preços e provoca alterações nas fontes de oferta e demanda de produtos.

Como pode-se notar, a ampla literatura sobre o impacto da volatilidade do câmbio no comércio internacional apresenta resultados distintos e inconclusivos. Como destaca Willett (1986), parte da dificuldade em se verificar a influência da volatilidade do câmbio no comércio pode ser devido a problemas estatísticos e a impossibilidade de determinados modelos estatísticos representarem adequadamente a incerteza do câmbio. De acordo com o autor, muitas vezes os impactos negativos da volatilidade cambial sobre o comércio encontrados em muitas pesquisas podem ser resultados isolados, sensíveis a escolha do período de tempo da amostra ou a especificação do modelo.

\section{DADOS E METODOLOGIA}

Este tópico objetiva explicitar o modelo econométrico a ser estimado e a especificação dos três métodos utilizados para mensuração da volatilidade da taxa real de câmbio e do impacto dessa volatilidade no comércio através da modelagem em painel.

\section{Modelo Gravitacional}

O modelo gravitacional estimado neste trabalho é análogo aos modelos gravitacionais oriundos da física, onde fatores como renda, distância e população influenciam o comércio internacional entre os países (Toda, 2012). Neste artigo busca-se estimar o modelo gravitacional com algumas das variáveis referidas acima acrescidas da volatilidade da taxa real de câmbio, de modo a estimar o efeito desta no comércio internacional.

Qual o impacto do câmbio no comércio e quais as medidas que podem ser colocadas em prática para que a volatilidade do câmbio seja controlada de forma a beneficiar as trocas comerciais? Antes de buscar quais medidas a serem adotadas, estima-se o impacto do câmbio através do modelo gravitacional abaixo para o período de 2001 a 2014 :

$$
\operatorname{lnExport}_{i j, t}=\beta_{1}+\beta_{2} \ln P I B_{i j, t}+\beta_{3} \operatorname{lnDist}_{i j, t}+\beta_{4} \operatorname{Vol}_{i j, t}+\beta_{5} T C_{i j, t}+W_{i t}
$$

Tem-se como variável explicada o logaritmo neperiano das exportações de cada país, onde as exportações são obtidas do WITS (World Integration Trade Solution), representando o comércio. Como variáveis explanatórias, temos $\ln P I B_{i j, t}$ como logaritmo neperiano do $\mathrm{PIB}^{1}$, lnDist ${ }_{i j, t}$ é o logaritmo neperiano da distância usado como proxy para os custos de transporte, $V o l_{i j, t}$ é a volatilidade da taxa real de câmbio e $T C_{i j, t}$ representa a volatilidade da taxa real de câmbio de um terceiro país no comércio entre o país i e j, $\beta_{1}$ é o termo de intercepto e, por último, $W_{i t}$ corresponde às variáveis não captadas pelo modelo.

Temos que:

$$
W_{i t}=\varepsilon_{i}+u_{i t}
$$

${ }^{1} \mathrm{PIB}$ a preços correntes encontrado no World Integration Trade Solution. 
Neste caso $\varepsilon_{i}$ representa o termo de erro individual de cada país utilizado no modelo, enquanto que $u_{i t}$ representa o termo de erro idiossincrático, sendo este, a soma dos erros de corte transversal e de séries temporais. Este é o modelo de dados em painel com efeitos aleatórios, que assume que os termos $\varepsilon_{i}$ e $u_{i t}$ possuem média zero, variância homocedástica

$$
\operatorname{lnExport}_{i j, t}=\beta_{1}+\beta_{2} \ln P I B_{i j, t}+\beta_{3} V_{i j, t}+\beta_{4} T C_{i j, t}+\alpha_{i}+u_{i t}
$$

Neste modelo a variável distância, a qual não varia no tempo, desaparece e $\alpha_{i}$ representa todos os efeitos observáveis e especifica uma média condicional estimável. Como nem todos os fatores grupo-específicos são observáveis, $\alpha_{i}$ deve estar correlacionado com os outros regressores. Se a hipótese de exogeneidade for correta o estimador de efeitos fixos é não viesado e $u_{i t}$ é não correlacionado com nenhuma das variáveis explicativas (Wooldridge, 2006). A escolha entre o modelo de efeitos fixos e aleatórios será efetuada a partir do resultado do teste de Wu-Hausman.

Para melhorar a eficiência dos coeficientes estimados do modelo, será utilizado um procedimento de Bootstrap com 2000 replicações. Conforme Johnston e Dinardo (2001), quando o pesquisador desconfia das estimativas dos parâmetros e a teoria assintótica fornece pistas muito fracas para a precisão de um estimador, o procedimento de Bootstrap pode ser aplicado, de forma a fornecer novas estimativas do erro padrão do estimador.

\section{VOLATILIDADE DA TAXA DE CÂMBIO REAL}

Para estimação da volatilidade da taxa real de câmbio canadense e mexicana em relação ao dólar estadunidense, será utilizada uma extensão do modelo generalizado de heterocedasticidade condicional (GARCH) introduzido por Bollerslev (1986), conhecido como IGARCH. Modelos autoregressivos de heterocedasticidade condicional (ARCH) (Engle, 1982) foram introduzidos antes dos modelos (GARCH) com $\mathrm{o}$ intuito de modelar o risco na teoria econômica e na teoria do portfólio, considerando que a variância e covariância variam ao longo do tempo, essa modelagem estatística de séries de tempo mostra-se eficiente na estimativa do risco, conforme Bueno (2008). Apesar dos modelos ARCH serem eficientes na modelagem e não possuem autocorrelação temporal e nem entre os indivíduos, ou seja, que $\varepsilon_{i}$ é um erro aleatório grupo-específico e que não está correlacionado com os regressores.

O modelo de efeitos fixos também será estimado, sendo que o mesmo é especificado da seguinte forma: da volatilidade, os modelos GARCH podem ser utilizados para descrever a volatilidade com menos parâmetros, tornando o modelo mais parcimonioso (Morettin, 2008). Os modelos GARCH são uma forma de dizer que a volatilidade depende de mais de uma defasagem, ou seja, que a volatilidade prevista não depende apenas do último período, mas também de períodos mais antigos (Pindyck e Rubinfeld, 2004).

Para estimar a volatilidade anual da taxa real bilateral de câmbio canadense e mexicana em relação ao dólar estadunidense, será utilizado o retorno mensal da taxa real de câmbio real para o período de março de 2000 a dezembro de 2014. A taxa real de câmbio foi calculada de acordo com a seguinte fórmula:

$$
R E R_{i j, t}=N E R_{i j, t}\left(\frac{C P I_{j, t}}{C P I_{i, t}}\right)
$$

sendo que $R E R_{i j, t}$ e $N E R_{i j, t}$ correspondem à taxa real e nominal de câmbio, respectivamente, do país i em relação ao país j no período t, e assim como $C P I_{j t}$ correspondem ao índice de preços ao consumidor (consumer price index) do país i e do país j para o período t. O índice de preços de cada país representa a variação de preços de uma cesta representativa de produtos consumida em cada país. No caso do México, a fonte dos dados provém do International Financial Statistics do fundo monetário internacional, no caso do Canadá a fonte de dados é o Statistics Canada enquanto que para os Estados Unidos, os dados provêm da Agência de Estatísticas de Trabalho estadunidense. A taxa nominal de câmbio assim como os índices de preços, foram extraídos do terminal Bloomberg.

Optou-se por utilizar o retorno da taxa real de câmbio, pois este apresenta estacionariedade no nível de significância de 1\% no teste Dickey-Fuller aumentado. Os retornos também possuem alguns fatos estilizados interessantes para a estimação dos modelos 
de séries temporais como: os retornos geralmente são não auto-correlacionados, o quadrado dos retornos são auto correlacionados apresentando uma correlação de uma defasagem que decai lentamente, e as séries de retornos apresentam agrupamentos de volatilidade no decorrer do tempo (Morettin, 2008).

O modelo utilizado na estimação da volatilidade do câmbio real foi o EWMA (exponentially weighted moving average) que pode ser formulado de forma equivalente através da modelagem GARCH tornando-se um IGARCH (Integrated GARCH) (Morettin, 2008). Esse modelo integrado (IGARCH) é caracterizado pela restrição de que a soma dos parâmetros deve ser igual a 1 (Bueno, 2008). Para o caso do México o modelo pode ser especificado da seguinte forma:

$$
\begin{aligned}
& \operatorname{rer}_{\text {mex }}=\emptyset_{4} \text { rer }_{t-4}-\theta_{1} a_{t-1}+a_{t} \\
& a_{t}=\sigma_{t}^{2} \varepsilon_{t} \\
& \sigma_{t}^{2}=\gamma \sigma_{t-1}^{2}+(1-\gamma) \operatorname{rer}_{t-1}^{2}
\end{aligned}
$$

sendo que $r e r_{\text {mex }}$ representa o retorno da taxa de câmbio real mexicana em relação ao dólar estadunidense, $\varnothing$ e $\theta$ representam parâmetros auto regressivos e de médias móveis, respectivamente, e $a_{t}$ representa o termo de erro no período t para o modelo ARMA(4,1). Conforme (7), $\sigma^{2}{ }_{t}$ é a variância condicionada à informação passada, enquanto que $\gamma e(1-\gamma)$ são parâmetros que somados devem ser iguais a 1 . Todos os parâmetros mostrados acima seguem a distribuição normal e são significativos ao nível de significância de $1 \%$.

Para o Canadá, o modelo que proporcionou melhor ajuste pode ser especificado da seguinte forma:

$$
\begin{gathered}
\text { lnrer }_{\text {can }}=\ln \left(1+\text { rer }_{\text {can }}\right) \\
\operatorname{lnrer}_{\text {can }}=\mu+a_{t} \\
a_{t}=\sigma_{t}^{2} \varepsilon_{t} \\
\sigma_{t}^{2}=\gamma \sigma_{t-1}^{2}+(1-\gamma) \text { lnrer }_{t-1}^{2}
\end{gathered}
$$

sendo que Inrer can representa o logaritmo neperiano do retorno da taxa de câmbio real canadense, $a_{t}$ representa o termo de erro no período t para a constante que é a média dos retornos no período. Assim como no modelo especificado para o México, $\sigma^{2}{ }_{t}$ é a variância condicionada à informação passada, enquanto $\gamma e(1-\gamma)$ são os parâmetros que somados devem ser igual a 1 .

Geralmente os modelos GARCH apresentam reversão a uma média de longo prazo da volatilidade, ou seja, existe uma volatilidade de longo prazo para alguns modelos GARCH. No caso dos modelos IGARCH essa volatilidade de longo prazo é inexistente e assume-se que esta segue um passeio aleatório, dado que a distribuição não condicional dos retornos não é estacionária, pois a soma dos parâmetros do modelo é igual a um (Alexander, 2005).

O gráfico 1 representa a volatilidade do retorno da taxa real de câmbio para o México e Canadá dos modelos descritos acima, em relação ao dólar estadunidense.

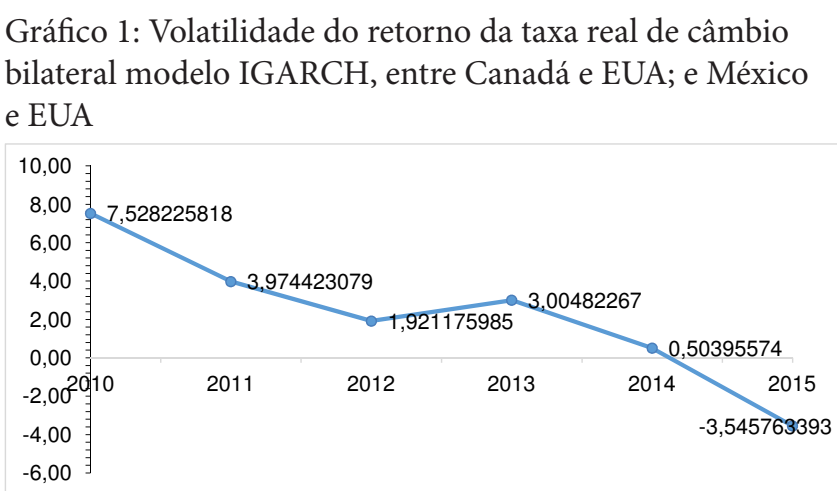

Fonte: Elaborado pelos autores.

No eixo vertical tem-se a variação do retorno da taxa real de câmbio (volatilidade) em relação ao período anterior, enquanto que no eixo horizontal encontra-se o período para o qual a volatilidade é estimada. Para anualizar a volatilidade calculada, foi feita uma média aritmética simples das estimativas de volatilidade a cada 12 meses.

Através do gráfico 1 , nota-se que a volatilidade da taxa real de câmbio para os anos de 2008 e 2009, aumentou significativamente se comparados aos demais períodos da série histórica. Este aumento acontece durante a crise do subprime nos Estados Unidos. Esse fato não é coincidência, já que um dos fatores que podem explicar esse aumento da volatilidade na taxa de câmbio real seriam alterações nas contas correntes dos países envolvidos, sendo que dentre os elementos para tal, tem-se a queda nas importações estadunidenses provenientes do México e Canadá ${ }^{2}$ Para se

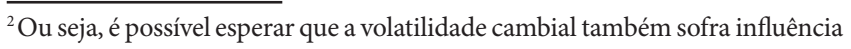


ter uma noção, durante a crise financeira o consumo nos Estados Unidos em 2008 aumentou somente 0,3\% em relação ao consumo de 2007, sendo esta a menor variação desde 1991, assim como a renda dos americanos aumentou somente $3,7 \%$ em relação a 2007 , sendo este resultado o menor desde 2003, além da queda de 4,9\% nas encomendas à indústria (Pinheiro, 2009).

Variações na renda, no consumo e nas encomendas à indústria podem influenciar as importações dos EUA provenientes dos seus parceiros comerciais. O gráfico 2 mostra as importações americanas vindas do Canadá e México de 2001 a 2015.

Gráfico 2: Importações estadunidenses provenientes do Canadá e México (em US\$ mil)

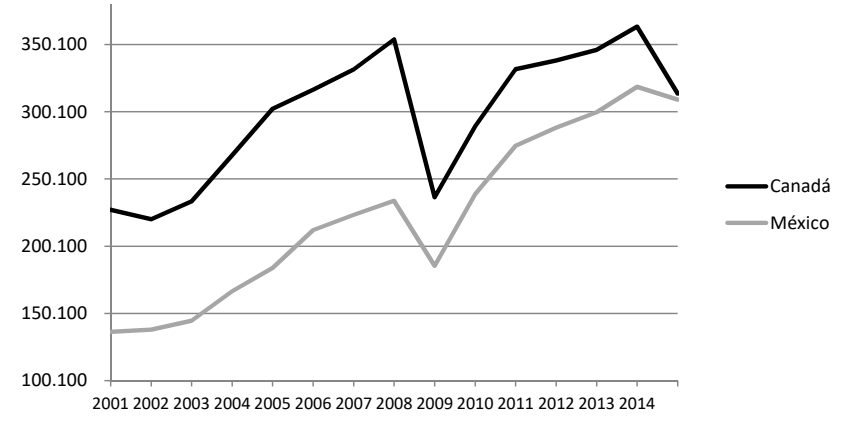

Fonte: Elaborado pelos autores com base em dados do Trade Map.

No eixo vertical tem-se o valor das importações, incluindo todos os tipos de produtos em mil dólares americanos, e no eixo horizontal o período em análise.

A redução nas importações estadunidenses por volta de 2008 reduziu a oferta de dólares americanos nas economias mexicanas e canadenses, depreciando o peso mexicano frente ao dólar americano (o dólar canadense manteve-se praticamente estável). Como a taxa de câmbio nominal influencia diretamente a taxa de câmbio real, houve, assim, uma depreciação da taxa de câmbio real do México. É razoável supor que a redução do nível de comércio do Canadá e México com os Estados Unidos no período de 2008 tenha reduzido a quantidade de divisas estadunidenses nesses países, pois, em média, aproximadamente $80 \%$ dos produtos exportados do Canadá são destinados para os Estados Unidos, e esse valor é de aproximadamente $82 \%$ para

da conta corrente ou comércio dos países, o que resulta em um problema de endogeneidade na estimação de modelos gravitacionais, a qual não será abordada neste artigo devido ao limitado número de observações da amostra disponível. Uma justificativa adicional para tal é que as exportações representam apenas um dos elementos que compõem a conta corrente. o México. A proporção das exportações desses países destinadas aos EUA foi calculada através de uma média aritmética simples do período de 2001 a 2015 dos dados obtidos do Trade Map (Trade Statistics for Intenational Business Development).

Portanto, o comércio desses países com os Estados Unidos é bastante significativo, e uma redução na demanda estadunidense por produtos mexicanos e canadenses, pode ter impactos significativos na taxa real e na volatilidade do câmbio destes países parceiros (gráfico 1), assim como em outras variáveis econômicas.

A outra medida de volatilidade é baseada em Peree e Steinherr (1989), a qual reflete as expectativas dos agentes baseadas em suas experiências passadas do máximo e mínimo valor da taxa de câmbio real bilateral. O cálculo pode ser assim especificado:

$$
V_{i j, t}=\frac{\max X_{i j, t-k}^{t}-\min X_{i j, t-k}^{t}}{\min X_{i j, t-k}^{t}}+\left[1+\frac{\left|X_{i j, t}-X_{i j, t}^{k}\right|}{X_{i j, t}^{k}}\right]^{2}
$$

sendo que k é o período medido em anos, $\max X_{i j, t-k}^{t}$ é o valor mais alto para a taxa real de câmbio nos k períodos (neste caso de 2001 a 2014) e $\min X_{i j, t-k}^{t}$ corresponde ao menor valor para a taxa de câmbio real bilateral no período $\mathrm{k}$ analisado. Por último, $X^{k}{ }_{i j, t}$ se refere à média da taxa de câmbio real para o período de estudo (2001 a 2014). O valor calculado acima corresponde a uma estimativa da taxa de câmbio de equilíbrio de longo prazo (Bittencourt, Larson e Thompson, 2007). No gráfico 3, a seguir, tem-se a volatilidade baseada em Peree e Steinherr.

Gráfico 3: Volatilidade da taxa real de câmbio bilateral metodologia Peree e Steinherr entre Canadá e EUA; e México e EUA

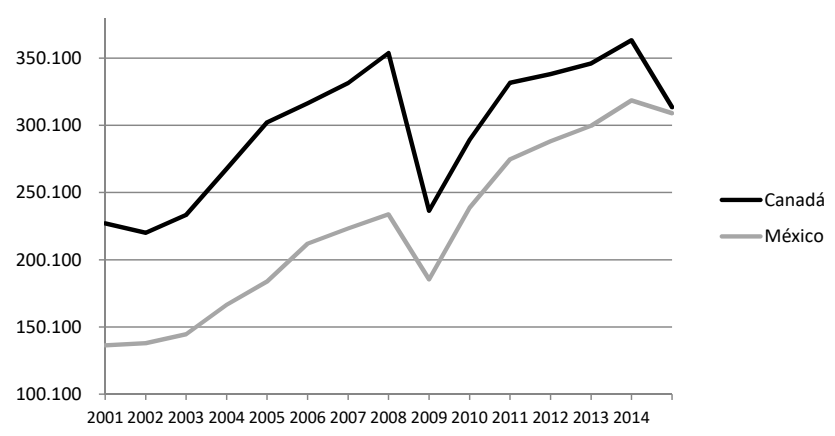

Fonte: Elaborado pelos autores. 
Analisando o gráfico, nota-se que a volatilidade baseada em Peree e Steinherr decresceu na década de 2010, e somente em 2014 teria voltado ao patamar de 2001 no caso do México, enquanto que pelo modelo IGARCH (gráfico 1) a volatilidade cresceu na década de 2010 e somente em 2014 teria voltado a patamares próximos ao de 2001. É interessante notar que volatilidade de Peree e Steinherr não mostra variações acentuadas durante as proximidades do ano de 2008 (crise do subprime), observando-se o contrário para o caso do modelo IGARCH, onde a volatilidade aumenta consideravelmente durante o período de crise.

Por último, foi calculado o desvio padrão móvel para o logaritmo da taxa real de câmbio do Canadá e México, conforme abaixo:

$$
S_{i j, t}=u_{i j, t}=\sqrt{\frac{\sum_{l=1}^{k}\left(x_{i j, t-l}-x_{i j, t}\right)^{2}}{k-1}}
$$

sendo que $x_{i j t-l}$ é o logaritmo natural da taxa de câmbio real bilateral EUA/México e EUA/Canadá. O desvio padrão móvel corresponde ao desvio da média da taxa real de câmbio para cada ano. No gráfico 4 tem-se a representação do comportamento da série de desvio padrão móvel.

Gráfico 4: Desvio Padrão Móvel para a taxa real de câmbio bilateral em relação ao dólar estadunidense

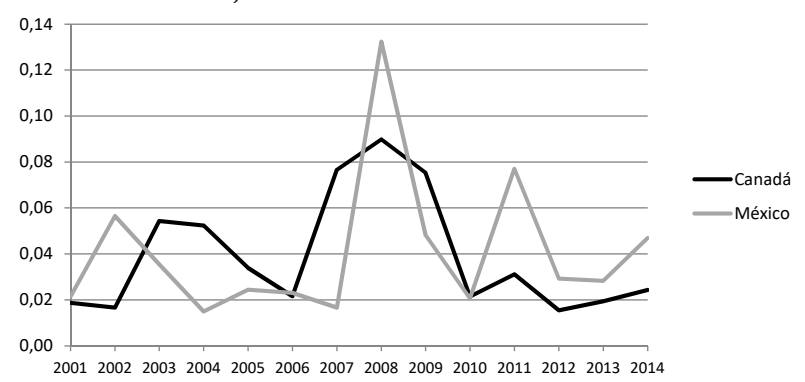

Fonte: Elaborado pelos autores.

Seguindo Bittencourt, Larson e Thompson (2007), a medida de volatilidade da ER real de um terceiro país, volatilidade "third country", $\left(\mathrm{u} 3_{\mathrm{ij}, t}\right)$ é dada por:

$$
\mathrm{TC}_{\mathrm{ij}, t}=\sum_{i \neq j} u_{\mathrm{ij}, t} w_{\mathrm{ij}, t} g+\sum_{j \neq i} u_{\mathrm{ji}, t} w_{\mathrm{ji}, t} g
$$

Onde $\mathrm{u}_{\mathrm{ij}, \mathrm{t}}\left(\mathrm{u}_{\mathrm{ji}, \mathrm{t}}\right)$ é a medida de volatilidade da ER real, seja ela a medida de Peree e Steinherr $\left(\mathrm{V}_{\mathrm{ij}, \mathrm{t}}\right)$ ou a medida de desvio padrão móvel $\left(\mathrm{S}_{\mathrm{ij}, \mathrm{t}}\right)$, definidas pelas equações (12) e (13), respectivamente; $g=1, \ldots$ , 8, onde 1 é para o setor agrícola; 2 para o setor de produtos químicos; 3 para o setor de alimentos; 4 para o setor de combustíveis; 5 para o setor de maquinaria e transportes; 6 para o setor de minérios e metais; 7 para o setor de têxteis; e 8 para o setor de manufaturas; $\mathrm{w}_{\mathrm{ij}, \mathrm{t}}{ }^{\mathrm{g}}$ e $\mathrm{w}_{\mathrm{ji}, \mathrm{t}}{ }^{\mathrm{g}}$ são as participações no comércio específicas a cada setor dos outros países. Espera-se que o sinal do coeficiente para o efeito "third country" no comércio seja positivo, como o obtido por Wei (1996). No entanto, para Dell'Ariccia (1999) esta correlação foi negativa e não significativa, e para Cho, Sheldon e McCorriston (2002) e Bittencourt, Larson e Thompson (2007) este coeficiente foi positivo e negativo para diferentes setores.

\section{RESULTADOS}

Estimados os modelos gravitacionais através da metodologia de dados em painel, encontrou-se resultados interessantes para os oito setores estudados e para o total do comércio do NAFTA. Para a agricultura, o modelo escolhido foi o de efeitos aleatórios, selecionado através do teste de Wu-Hausman. No modelo para a agricultura os parâmetros como PIB, distância e volatilidade do câmbio foram estatisticamente significativos a $1 \%$ para a metodologia de volatilidade IGARCH e apresentaram o sinal esperado de acordo com a teoria, indo no mesmo sentido dos resultados encontrados por Campos e Bittencourt (2014), Toda (2012) e Bittencourt, Larson e Thompson (2007) para este setor no comércio entre Brasil e seus parceiros. Bahmani-Oskooee e Scott (2009) também encontram impactos negativos e significativos para este setor no comércio entre Estados Unidos e México utilizando um modelo de correção de erros.

No entanto, o efeito terceiro país, ou third country (TC), não pode ser considerado estatisticamente diferente de zero. Para as demais medidas de volatilidade Peree e Steinherr e desvio padrão móvel os coeficientes não foram significativos, ao contrário dos trabalhos de Bittencourt, Larson e Thompson (2007) e Toda (2012), que encontraram coeficientes estatisticamente significativos para essas medidas de volatilidade e Bittencourt, Larson e Thompson (2007) que encontrou coeficientes significativos para o efeito TC. A tabela 1 abaixo sintetiza os resultados. 
Tabela 1: Impacto da Volatilidade da Taxa Real de Câmbio na Agricultura (NAFTA)

\begin{tabular}{|c|c|c|c|}
\hline \multirow{2}{*}{$\begin{array}{l}\text { Agricultura } \\
\text { Variável }\end{array}$} & \multicolumn{3}{|c|}{ Medidas de Volatilidade } \\
\hline & IGARCH (RE) & $\mathrm{P} \& \mathrm{~S}(\mathrm{RE})$ & $\mathrm{SD}(\mathrm{RE})$ \\
\hline \multirow[t]{2}{*}{ PIB } & $0,5329 * * *$ & $0,3855^{* * *}$ & $0,4569 * * *$ \\
\hline & $(0,1252)$ & $(0,1030)$ & $(0,1230)$ \\
\hline \multirow[t]{2}{*}{ Distância } & $-1,8624 * * *$ & $-1,9025^{* * *}$ & $-1,8911 * * *$ \\
\hline & $(0,0850)$ & $(0,1068)$ & $(0,1077)$ \\
\hline \multirow[t]{2}{*}{ Volatilidade } & $-10,2309 * * *$ & $-0,1678$ & $-0,7758$ \\
\hline & $(3,3831)$ & $(0,2394)$ & $(1,4333)$ \\
\hline \multirow[t]{2}{*}{ Terceiro País } & $-0,06197$ & $-0,0936$ & $-0,0686$ \\
\hline & $(0,2281)$ & $(0,2705)$ & $(0,2903)$ \\
\hline \multicolumn{2}{|c|}{ Observações $=84 ; \mathrm{t}=14 ; \mathrm{i}=3$} & & \\
\hline
\end{tabular}

Fonte: WITS. Cálculos dos autores.

Nota: os valores entre parêntesis correspondem ao desvio padrão encontrado por bootstrap.

$\left.{ }^{*}\right)$ Estatisticamente significativo ao nível de $10 \%$; $\left.{ }^{* *}\right)$

Estatisticamente significativo ao nível de 5\%; $\left.{ }^{(* *}\right)$

Estatisticamente significativo ao nível de $1 \%$.

(RE) modelo de efeitos aleatórios; (FE) modelo de efeitos fixos.

No caso da volatilidade do câmbio mensurada pela medida IGARCH, pode-se interpretar o coeficiente como se uma variação de $10 \%$ ocorresse na volatilidade da taxa real de câmbio, o impacto seria de uma variação de $2,58 \%{ }^{3}$ nas exportações de produtos agrícolas dos países do NAFTA. No caso do PIB, um aumento na renda de $10 \%$ dos países do bloco, aumentaria as exportações de bens agrícolas entre os países membros em 5,32\%, sendo este o caso, para o modelo com a medida de volatilidade especificada na forma de um IGARCH. A variável distância se mostra negativamente relacionada com o comércio nos três modelos estimados, como esperado pela teoria.
Tabela 2: Impacto da Volatilidade da Taxa Real de Câmbio no Setor Químico (NAFTA)

\begin{tabular}{l|c|c|c}
\hline Químico & \multicolumn{3}{|c}{ Medidas de Volatilidade } \\
\hline Variável & IGARCH (FE) & P\&S (FE) & SD (FE) \\
\hline PIB & $1,672 * * *$ & $1,5969 * * *$ & $1,6529 * * *$ \\
\hline & $(0,1539)$ & $(0,1404)$ & $(0,1505)$ \\
\hline Distância & - & - & - \\
\hline & - & - & - \\
\hline Volatilidade & $-2,2118$ & $-0,1279$ & $-0,6904$ \\
\hline & $(2,8728)$ & $(0,1664)$ & $(0,8541)$ \\
\hline Terceiro País & 0,0017 & $-0,0153$ & 0,0046 \\
\hline \multicolumn{4}{l}{} \\
\hline \multicolumn{2}{l|}{ Observações $=84 ; \mathrm{t}=14 ; \mathrm{i}=3$} & $(0,0711)$ & $(0,0731)$ \\
\hline
\end{tabular}

Fonte: WITS. Cálculos dos autores.

Nota: os valores entre parêntesis correspondem ao desvio padrão encontrado por bootstrap.

${ }^{*}$ ) Estatisticamente significativo ao nível de 10\%; $\left.{ }^{* *}\right)$

Estatisticamente significativo ao nível de 5\%; $\left.{ }^{(* *}\right)$

Estatisticamente significativo ao nível de $1 \%$.

(RE) modelo de efeitos aleatórios; (FE) modelo de efeitos fixos.

Para o setor químico (tabela 2) o coeficiente do PIB é estatisticamente significativo e apresenta o sinal esperado teoricamente. Como o modelo escolhido para este setor é o de efeitos fixos, a variável distância foi eliminada do modelo por ser invariante no tempo. A volatilidade da taxa real de câmbio e o efeito third country (volatilidade da taxa real de câmbio de um terceiro país) não são significativos indo de encontro com o resultado encontrado por Toda (2012) e contrastando com Bittencourt, Larson e Thompson (2007) e Campos e Bittencourt (2014), os quais encontraram coeficientes estatisticamente significativos para este setor no comércio entre Brasil e seus parceiros.

\footnotetext{
${ }^{3}$ Para a interpretação do impacto de uma redução na volatilidade multiplicou-se o valor do coeficiente estimado pelo modelo IGARCH pela média amostral da volatilidade, sendo esta igual a 0,0253 .
} 
Tabela 3: Impacto da Volatilidade da Taxa Real de Câmbio no Setor de Alimentos (NAFTA)

\begin{tabular}{|c|c|c|c|}
\hline Alimentos & \multicolumn{3}{|c|}{ Medidas de Volatilidade } \\
\hline Variável & IGARCH (FE) & $\mathrm{P} \& \mathrm{~S}(\mathrm{FE})$ & $\mathrm{SD}(\mathrm{FE})$ \\
\hline \multirow[t]{2}{*}{ PIB } & $1,7838 * * *$ & $1,8492 * * *$ & $1,7790 * * *$ \\
\hline & $(0,1288)$ & $(0,1275)$ & $(0,1264)$ \\
\hline \multirow[t]{2}{*}{ Distância } & - & - & - \\
\hline & - & - & - \\
\hline \multirow[t]{2}{*}{ Volatilidade } & $-0,4816$ & 0,1493 & $-0,2920$ \\
\hline & $(2,3056)$ & $(0,1156)$ & $(0,7286)$ \\
\hline \multirow[t]{2}{*}{ Terceiro País } & $-0,0006$ & 0,0157 & 0,0011 \\
\hline & $(0,0619)$ & $(0,0655)$ & $(0,0648)$ \\
\hline \multicolumn{2}{|c|}{ Observações $=84 ; \mathrm{t}=14 ; \mathrm{i}=3$} & & \\
\hline
\end{tabular}

Fonte: WITS. Cálculos dos autores.

Nota: os valores entre parêntesis correspondem ao desvio padrão encontrado por bootstrap.

$\left.{ }^{*}\right)$ Estatisticamente significativo ao nível de $10 \%$; $\left.{ }^{* *}\right)$

Estatisticamente significativo ao nível de 5\%; $\left({ }^{* *}\right)$

Estatisticamente significativo ao nível de $1 \%$.

(RE) modelo de efeitos aleatórios; (FE) modelo de efeitos fixos.

Para o setor de alimentos (tabela 3) nenhuma das medidas de volatilidade mostrou-se significativa para o comércio desses bens.

Tabela 4: Impacto da Volatilidade da Taxa Real de Câmbio no Setor de Combustíveis (NAFTA)

\begin{tabular}{|c|c|c|c|}
\hline Combustíveis & \multicolumn{3}{|c|}{ Medidas de Volatilidade } \\
\hline Variável & IGARCH (RE) & $\mathrm{P} \& \mathrm{~S}(\mathrm{RE})$ & $\mathrm{SD}(\mathrm{RE})$ \\
\hline \multirow[t]{2}{*}{ PIB } & $2,0226 * * *$ & $1,9736 * * *$ & $2,0857 * * *$ \\
\hline & $(0,2938)$ & $(0,3687)$ & $(0,3568)$ \\
\hline \multirow[t]{2}{*}{ Distância } & $-0,9113 * * *$ & $-0,9130 * * *$ & $-0,8820 * * *$ \\
\hline & $(0,1272)$ & $(0,1355)$ & $(0,1288)$ \\
\hline \multirow[t]{2}{*}{ Volatilidade } & 9,0521 & $-0,2634$ & 2,0688 \\
\hline & $(5,6046)$ & $(0,2847)$ & $(1,8678)$ \\
\hline \multirow[t]{2}{*}{ Terceiro País } & 0,0626 & 0,0431 & 0,0566 \\
\hline & $(0,3100)$ & $(0,4424)$ & $(0,3885)$ \\
\hline \multicolumn{2}{|c|}{ Observações $=84 ; \mathrm{t}=14 ; \mathrm{i}=3$} & & \\
\hline
\end{tabular}

Fonte: WITS. Cálculos dos autores.

Nota: os valores entre parêntesis correspondem ao desvio padrão encontrado por bootstrap.

$\left.{ }^{*}\right)$ Estatisticamente significativo ao nível de $10 \%$; $\left(^{* *}\right)$

Estatisticamente significativo ao nível de $5 \%$; $\left(^{* * *}\right)$

Estatisticamente significativo ao nível de $1 \%$.

(RE) modelo de efeitos aleatórios; (FE) modelo de efeitos fixos.

A tabela 4 mostra os parâmetros do modelo estimados para o setor de combustíveis. Neste setor também se nota a ausência de influência de variações do câmbio nas exportações de combustíveis entre os países do bloco. Nenhuma das três medidas de volatilidade se mostrou significativa, no entanto, PIB e distância podem impactar de forma ativa no comércio desses países.

Tabela 5: Impacto da Volatilidade da Taxa Real de Câmbio no Setor de Maquinaria e Transportes (NAFTA)

\begin{tabular}{|c|c|c|c|}
\hline \multirow{2}{*}{$\begin{array}{l}\text { Maquinaria e Trans. } \\
\text { Variável }\end{array}$} & \multicolumn{3}{|c|}{ Medidas de Volatilidade } \\
\hline & IGARCH (RE) & $\mathrm{P} \& \mathrm{~S}(\mathrm{RE})$ & $\mathrm{SD}(\mathrm{RE})$ \\
\hline \multirow[t]{2}{*}{ PIB } & $1,0276^{* * *}$ & $1,0324 * * *$ & $0,9687 * * *$ \\
\hline & $(0,2313)$ & $(0,2433)$ & $(0,2177)$ \\
\hline \multirow[t]{2}{*}{ Distância } & $-0,7365 * * *$ & $-0,7435 * * *$ & $-0,7617 * * *$ \\
\hline & $(0,0982)$ & $(0,1014)$ & $(0,1039)$ \\
\hline \multirow[t]{2}{*}{ Volatilidade } & $-8,2047 *$ & 0,1577 & $-1,5604$ \\
\hline & $(4,7631)$ & $(0,2545)$ & $(1,7593)$ \\
\hline \multirow[t]{2}{*}{ Terceiro País } & $-0,0870$ & $-0,0805$ & $-0,0862$ \\
\hline & $(0,3429)$ & $(0,3826)$ & $(0,3773)$ \\
\hline \multicolumn{2}{|c|}{ Observações $=84 ; \mathrm{t}=14 ; \mathrm{i}=3$} & & \\
\hline
\end{tabular}

Fonte: WITS. Cálculos dos autores.

Nota: os valores entre parêntesis correspondem ao desvio padrão encontrado por bootstrap.

$\left.{ }^{*}\right)$ Estatisticamente significativo ao nível de $10 \%$; $\left(^{* *}\right)$ Estatisticamente significativo ao nível de 5\%; ${ }^{* * *}$ ) Estatisticamente significativo ao nível de $1 \%$.

(RE) modelo de efeitos aleatórios; (FE) modelo de efeitos fixos.

A tabela 5 sintetiza os resultados para o setor de maquinaria e transportes. Diferentemente dos setores químicos, alimentos e combustíveis, o setor de maquinaria e transportes é estatisticamente influenciado negativamente pela volatilidade da taxa real de câmbio para o caso da medida IGARCH, indo de encontro com o resultado encontrado por Campos e Bittencourt (2014) para este setor no comércio do Brasil e seus parceiros. Uma redução de $10 \%$ na volatilidade cambial pode elevar as exportações de maquinários na integração regional do NAFTA em 2,07\%. Os coeficientes para o PIB e distância são estatisticamente significativos ao nível de $1 \%$, enquanto a volatilidade é significativa somente ao nível de $10 \%$. 
Tabela 6: Impacto da Volatilidade da Taxa Real de Câmbio no Setor de Minérios e Metais (NAFTA)

\begin{tabular}{l|c|c|c}
\hline Minérios e Metais & \multicolumn{3}{|c}{ Medidas de Volatilidade } \\
\hline Variável & IGARCH (FE) & P\&S (RE) & SD (FE) \\
\hline PIB & $1,8884 * * *$ & $1,2771^{* * *}$ & $1,8903 * * *$ \\
\hline & $(0,2236)$ & $(0,4325)$ & $(0,2162)$ \\
\hline Distância & - & $-1,0145 * * *$ & - \\
\hline & - & $(0,1467)$ & - \\
\hline Volatilidade & 0,0784 & $-0,1264$ & 0,3157 \\
\hline & $(4,3855)$ & $(0,3376)$ & $(1,2390)$ \\
\hline Terceiro País & $-0,0912$ & $-0,1972$ & $-0,0936$ \\
\hline & $(0,1886)$ & $(0,4394)$ & $(0,1826)$ \\
\hline
\end{tabular}

Observações $=84 ; \mathrm{t}=14 ; \mathrm{i}=3$

Fonte: WITS. Cálculos dos autores.

Nota: os valores entre parêntesis correspondem ao desvio padrão encontrado por bootstrap.

$\left.{ }^{*}\right)$ Estatisticamente significativo ao nível de $10 \%$; $\left.{ }^{* *}\right)$

Estatisticamente significativo ao nível de 5\%; ${ }^{* * *}$ )

Estatisticamente significativo ao nível de $1 \%$.

(RE) modelo de efeitos aleatórios; (FE) modelo de efeitos fixos.

Para o setor de minérios e metais, nenhuma das três medidas de volatilidade impacta o comércio nesse setor, resultado diferente do encontrado por Bittencourt, Larson e Thompson (2007), Toda (2012) e Campos e Bittencourt (2014) que encontraram coeficientes significativos para o comércio entre Brasil e seus parceiros. Todos os coeficientes apresentaram o sinal esperado, sendo que a distância impacta negativamente o comércio do setor e o PIB influencia positivamente.

Tabela 7: Impacto da Volatilidade da Taxa Real de Câmbio no Setor de Produtos Têxteis (NAFTA)

\begin{tabular}{|c|c|c|c|}
\hline Têxteis & \multicolumn{3}{|c|}{ Medidas de Volatilidade } \\
\hline Variável & IGARCH (RE) & $\mathrm{P} \& \mathrm{~S}(\mathrm{FE})$ & $\mathrm{SD}(\mathrm{RE})$ \\
\hline \multirow[t]{2}{*}{ PIB } & $0,2404 * *$ & 0,0207 & 0,1600 \\
\hline & $(0,1118)$ & $(0,0972)$ & $(0,1319)$ \\
\hline \multirow[t]{2}{*}{ Distância } & $-1,1635^{* * *}$ & - & $-1,1941$ \\
\hline & $(0,1971)$ & - & $(0,2367)$ \\
\hline \multirow[t]{2}{*}{ Volatilidade } & $-12,1984 * * *$ & $-0,1988$ & $-1,4178$ \\
\hline & $(3,6819)$ & $(0,1337)$ & $(2,0186)$ \\
\hline \multirow[t]{2}{*}{ Terceiro País } & 0,0019 & $-0,0197$ & $-0,0036$ \\
\hline & $(0,4010)$ & $(0,0737)$ & $(0,4727)$ \\
\hline \multicolumn{2}{|c|}{ Observações $=84 ; \mathrm{t}=14 ; \mathrm{i}=3$} & & \\
\hline
\end{tabular}

Fonte: WITS. Cálculos dos autores.

Nota: os valores entre parêntesis correspondem ao desvio padrão encontrado por bootstrap.

$\left({ }^{*}\right)$ Estatisticamente significativo ao nível de $10 \%$; $\left({ }^{*}\right)$

Estatisticamente significativo ao nível de 5\%; ${ }^{* * *}$ )

Estatisticamente significativo ao nível de $1 \%$.

(RE) modelo de efeitos aleatórios; (FE) modelo de efeitos fixos.
Para o setor de têxteis, na estimação utilizando a medida de volatilidade IGARCH todos os parâmetros, exceto o efeito third country, se mostraram estatisticamente significativos. Para esse setor, uma redução de $10 \%$ na volatilidade pode resultar em uma elevação de 3,08\% nas exportações de produtos têxteis dentro do NAFTA. O impacto negativo da volatilidade cambial sobre o comércio de produtos têxteis também foi encontrado por Bahmani-Oskooee e Scott (2009) no comércio entre Estados Unidos e México no período de 1962 a 2004.

Tabela 8: Impacto da Volatilidade da Taxa Real de Câmbio no Setor de Produtos Manufaturados (NAFTA)

\begin{tabular}{|c|c|c|c|}
\hline \multirow{2}{*}{$\begin{array}{l}\text { Manufaturas } \\
\text { Variável }\end{array}$} & \multicolumn{3}{|c|}{ Medidas de Volatilidade } \\
\hline & IGARCH (FE) & $\mathrm{P} \& \mathrm{~S}(\mathrm{FE})$ & $\mathrm{SD}(\mathrm{FE})$ \\
\hline \multirow[t]{2}{*}{ PIB } & $1,2051 * * *$ & $1,2027 * * *$ & $1,1399 * * *$ \\
\hline & $(0,1202)$ & $(0,1178)$ & $(0,1146)$ \\
\hline \multirow[t]{2}{*}{ Distância } & - & - & - \\
\hline & - & - & - \\
\hline \multirow[t]{2}{*}{ Volatilidade } & $-8,0974 * * *$ & 0,1229 & $-1,4231^{*}$ \\
\hline & $(2,8149)$ & $(0,1546)$ & $(0,7501)$ \\
\hline \multirow[t]{2}{*}{ Terceiro País } & $-0,0583$ & $-0,0541$ & $-0,0567$ \\
\hline & $(0,0638)$ & $(0,0637)$ & $(0,0671)$ \\
\hline \multicolumn{2}{|c|}{ Observações $=84 ; \mathrm{t}=14 ; \mathrm{i}=3$} & & \\
\hline
\end{tabular}

Fonte: WITS. Cálculos dos autores.

Nota: os valores entre parêntesis correspondem ao desvio padrão encontrado por bootstrap.

${ }^{*}$ ) Estatisticamente significativo ao nível de $10 \%$; $\left(^{* *}\right)$

Estatisticamente significativo ao nível de 5\%; $\left(^{* * *}\right)$

Estatisticamente significativo ao nível de $1 \%$.

(RE) modelo de efeitos aleatórios; (FE) modelo de efeitos fixos.

Na tabela 8 seguem os resultados dos modelos estimados para o setor de manufaturas. No caso das manufaturas, a medida de volatilidade IGARCH mostra-se significativa ao nível de $1 \%$, enquanto o Desvio Padrão Móvel (SD) foi significativo a 10\%, revelando que uma redução de $10 \%$ na volatilidade da taxa real de câmbio pode resultar em um aumento de 2,04\% das exportações no caso do modelo IGARCH e para o modelo SD essa redução elevaria em $0,55 \%{ }^{4}$ as exportações de bens manufaturados no NAFTA. Bittencourt, Larson e Thompson (2007) e Toda (2012) também encontraram coeficientes significativos para

\footnotetext{
${ }^{4}$ Para a interpretação do impacto de uma redução na volatilidade multiplicou-se o valor do coeficiente estimado pelo modelo SD pela média amostral da volatilidade, sendo esta igual a 0,0389 .
} 
o setor de manufaturados no comércio entre Brasil e seus parceiros.

Tabela 9: Impacto da Volatilidade da Taxa Real de Câmbio no Total do Comércio dos Países do NAFTA

\begin{tabular}{l|c|c|c}
\hline Total & \multicolumn{3}{|c}{ Medidas de Volatilidade } \\
\hline Variável & IGARCH (RE) & P\&S (RE) & SD (FE) \\
\hline PIB & $1,2791^{* * *}$ & $1,2411^{* * *}$ & $1,2917^{* * *}$ \\
\hline & $(0,0588)$ & $(0,0617)$ & $(0,0638)$ \\
\hline Distância & $-0,9576^{* * *}$ & $-0,9689 * * *$ & - \\
\hline & $(0,0341)$ & $(0,0347)$ & - \\
\hline Volatilidade & $-3,9203 * * *$ & $-0,0241$ & $-0,4953$ \\
\hline & $(1,4204)$ & $(0,0665)$ & $(0,4036)$ \\
\hline Terceiro País & $-0,0286$ & $-0,0361$ & $-0,0203$ \\
\hline & $(0,0354)$ & $(0,0361)$ & $(0,0351)$ \\
\hline
\end{tabular}

Observações $=84 ; \mathrm{t}=14 ; \mathrm{i}=3$

Fonte: WITS. Cálculos dos autores.

Nota: os valores entre parêntesis correspondem ao desvio padrão encontrado por bootstrap.

$\left.{ }^{*}\right)$ Estatisticamente significativo ao nível de 10\%; $\left.{ }^{* *}\right)$

Estatisticamente significativo ao nível de $5 \% ;\left({ }^{* * *}\right)$

Estatisticamente significativo ao nível de $1 \%$.

(RE) modelo de efeitos aleatórios; (FE) modelo de efeitos fixos.

Para o total do comércio dos países do NAFTA, a medida de volatilidade IGARCH mostrou-se estatisticamente significativa ao nível de $1 \%$. Neste caso uma redução de $10 \%$ na volatilidade da taxa real de câmbio pode impactar em um aumento de aproximadamente $0,99 \%$ no comércio total entre os países do NAFTA. No modelo em que foi utilizada a medida de volatilidade IGARCH, os coeficientes do PIB e da distância assumem valores significativos e com os sinais esperados. No caso do PIB, uma variação de $10 \%$ na renda pode resultar em uma variação de aproximadamente $12,79 \%$ nas exportações dentro do bloco. Toda (2012) e Bittencourt, Larson e Thompson (2007), também encontraram coeficientes significativos e negativos para o impacto da volatilidade da taxa real de câmbio no total do comércio entre Brasil e seus parceiros, assim como Bahmani-Oskooee e Scott (2009) e Augustine (1995) observaram que incrementos na volatilidade cambial possui impactos negativos no total do comércio entre Estados Unidos e México e nas exportações americanas.

No entanto, apesar dos resultados encontrados mostrarem o impacto negativo da volatilidade, a medida de volatilidade Peree-Steinherr não foi significativa para nenhum setor do presente artigo, enquanto a medida Desvio Padrão Móvel foi significativa ao nível de $10 \%$ somente para o setor de manufaturados, o que difere dos resultados encontrados por Bittencourt, Larson e Thompson (2007) e Toda (2012) para estas medidas de volatilidade. Estas diferenças nos resultados entre o presente estudo e os demais é um motivo para frisar o alerta proposto por Willett (1986) e Clark et al (2004) de que a medida de volatilidade e o período de tempo podem influenciar significativamente nos resultados encontrados quanto ao impacto da volatilidade do câmbio no comércio internacional. Por este motivo esta pesquisa utilizou três medidas de volatilidade, com o objetivo de compará-las e obter resultados mais amplos.

\section{DISCUSSÃO}

Dos modelos gravitacionais estimados para cada setor, somente os setores agricultura, maquinaria e transportes, têxteis e manufaturas apresentaram significância estatística para a medida de volatilidade IGARCH. Para a medidas de volatilidade Peree e Steinherr, nenhum dos setores apresentaram coeficientes significativos, enquanto que somente para o setor de manufaturados o coeficiente do Desvio Padrão Móvel mostrou-se estatisticamente diferente de zero. PIB e distância apresentaram significância estatística para todos os setores, inclusive para o total do comércio. A medida de volatilidade de um terceiro país (third country) não apresentou significância estatística para nenhum dos setores e nem para o total do comércio. Os resultados apresentados para o total do comércio mostram que a volatilidade calculada pela medida IGARCH influencia negativamente as exportações entre os países do NAFTA, e que este comércio é influenciado também positivamente pela renda e negativamente pela distância entre os países.

Buscando entender porque as exportações de alguns setores estão mais suscetíveis à volatilidade do câmbio, o modelo de hysteresis de Baldwin e Krugman explica que setores que necessitam de uma grande quantidade de investimentos sofrem menos se comparados a setores que necessitam de uma menor quantidade de recursos. Outros fatores também podem ser responsáveis pela diferença de impacto da volatilidade da taxa real de câmbio entre os setores, como grau de substitubilidade dos produtos e se os bens são duráveis ou não (Bittencourt, Larson e Thompson, 2007). Os setores relacionados à combustíveis, metais 
e minérios, alimentos e químico não sofrem influência da volatilidade cambial, nesse caso, podemos dizer que o setor de alimentos é o único que necessita de baixos investimentos comparativamente aos demais, porém, é um setor que possui um grau muito alto de substitubilidade, dessa forma, variações muito bruscas na taxa de câmbio podem fazer com que alimentos provenientes dos mercados externos sejam facilmente substituídos por alimentos produzidos no mercado doméstico. Os setores de metais e minérios, químicos e combustíveis, normalmente necessitam de elevados investimentos, neste caso, estão de acordo com a teoria por não sofrerem impacto da volatilidade do câmbio.

O setor agrícola, de maquinaria e transportes, têxteis e manufaturas, são suscetíveis a variações na volatilidade da taxa de câmbio, no entanto, podemos considerar somente os setores agrícolas e têxteis como setores de baixo investimento e que de acordo com o modelo de hysteresis tenderiam a sofrer com a volatilidade do câmbio. Então, por que os setores de manufaturas e de maquinaria e transportes também são impactados negativamente pela taxa de câmbio? Pode-se dizer que os bens manufaturados externos são bens facilmente substituídos pelo bem doméstico, no entanto, maquinaria e transporte não possuem tal vantagem. Dessa forma, pode-se explicar o impacto da volatilidade cambial sofrido pelo setor de maquinários, pelo fato de até mesmo em países desenvolvidos o comércio de bens de capital se tornar difícil em períodos de grande incerteza cambial, assim, essa incerteza tende a influenciar negativamente no comércio desses produtos.

\section{CONSIDERAÇÕES FINAIS}

De modo geral, na busca para explicar o impacto da volatilidade cambial no comércio setorial dos países do NAFTA, as estimativas do presente estudo trouxeram resultados satisfatórios do ponto de vista teórico e estatístico, sendo que, de oito setores estudados quatro apresentaram coeficientes de volatilidade significativos ao contrário dos quatro restantes. Pode-se afirmar que, para o total do comércio entre os países do NAFTA no período de 2001 a 2014, a volatilidade cambial foi estatisticamente significativa de modo a impactar de forma negativa as exportações do bloco regional, indo no sentido contrário aos resultados encontrados por Asseery e Peel (1991), no qual a volatilidade impactou positivamente o comércio e no mesmo sentido de Bahmani-Oskooee e Scott (2009), Augustine (1995), Toda (2012), Campos e Bittencourt (2014), Bittencourt, Larson e Thompson (2007) e Chowdhury (1993) que observaram influência negativa. Uma explicação possível para o impacto negativo da volatilidade cambial no comércio internacional pode ser atribuída à aversão ao risco dos agentes (Clark et al, 2004), onde em períodos de grande incerteza estes preferem reduzir suas atividades, com o objetivo de evitar a exposição ao risco, em lugar de tentar obter potenciais ganhos com as fortes oscilações do câmbio (Bahmani-Oskooee e Scott, 2009).

Dessa forma, políticas econômicas que objetivem a redução da volatilidade cambial, podem auxiliar no crescimento do nível de emprego e renda e na redução da incerteza dos agentes. Portanto, este estudo buscou explorar as relações entre volatilidade cambial e comércio internacional, sendo que novas medidas de volatilidade e novos estudos podem ser feitos para reforçar ou contrastar os resultados obtidos com esta pesquisa, para que de forma produtiva auxilie na tomada de decisões.

\section{AGRADECIMENTOS}

Os autores gostariam de agradecer o apoio financeiro à pesquisa realizada junto ao Conselho Nacional de Desenvolvimento Científico e Tecnológico (CNPq) e todo o apoio dos membros do Núcleo de Economia Internacional e Desenvolvimento Econômico (NEIDE - UFPR/CNPq).

\section{REFERÊNCIAS}

Alexander, C. Modelos de Mercado: Um Guia para Análise de Informações Financeiras/Carol Alexander; tradução José Carlos de Souza Santos. São Paulo: Bolsa de Mercadorias e Futuros, 2005.

Anderson, J.E.; Van Wincoop, E. Trade costs. Journal of Economic Literature, v. 42, n. 3, p. 691-751, 2004.

Augustine, C. A. The Effects of Exchange-Rate Volatility on U.S. Exports: An Empirical Investigation. Southern Economic Journal, Texas, vol.62, n. 1, pp. 34-43, jul. 1995.

Asseery, A., and Peel, D. A. The Effects of Exchange Rate Volatility on Exports. Economics Letters, 37, 173-177. 1991. 
Bahmani-Oskooee, M. e Scott, W. H. The Effects of Exchange-Rate Volatilitiy on Commodity Trade between the United states and Mexico. Southern Economic Journal, vol.75, n 4, pp1019-1044. 2009.

Bittencourt, M. V.L.; Larson, D. W. e Thompson, S. R. Impactos da volatilidade da taxa de câmbio no comércio setorial do Mercosul. Estudos Econômicos, vol.37 no.4, São Paulo, Oct./Dec. 2007.

Bollerslev, T. Generalized autoregressive conditional heteroscedasticity. Journal of Econometrics, v.31, n.3, 307-327, 1986.

Bueno, Rodrigo De Losso da Silveira. Econometria de Séries Temporais. São Paulo: Cengage Learning, 2008.

Campos, A. C.; Bittencourt, G. M. Efeitos da instabilidade da taxa/ de câmbio no comércio setorial entre Brasil e seus principais parceiros comerciais. Economia Aplicada, Vol. 18. n.4. Ribeirão Preto. 2014.

Carmo, A. S. S.; Bittencourt, M. V. L. O comércio intraindustrial entre Brasil e os países da OCDE: decomposição e análise de seus determinantes. Revista Análise Econômica, 2013.

Cheng, H.; Wall, H.J. Controlling for Heterogeneity in Gravity Models of Trade and Integration. Federal Bank of St. Louis Review, 87(1), pp. 49-63, January/February 2005.

Cho, G., Sheldon, I. M. and McCorriston, S. Exchange rate uncertainty and agricultural trade. American Journal of Agricultural Economics 84 (4), 932-942, 2002.

Chowdhury, A. R. Does Exchange Rate Voaltility Depress Trade Flows? Evidence from Error-Correction Models. The Review of Economics and Statistics, vol. 75, n. 4, pp. 700706, nov. 1993.

Clark,P.; Tamirisa,N.; Wei,S.J.; Sadikov,A; Zeng,L. Exchange Rate Volatility and TradeFlows - Some New Evidence. International Monetary Fund, 2004.

Dell'Ariccia, G. Exchange rate fluctuations and trade flows. IMF Staff Papers 46, 293-314, 1999.

Engle, R. Autoregressive conditional heteroscedasticity with estimates of the variance of United Kingdom inflation. Econometrica, v.50, n.4, 987-1007, 1982.

Franke, G. Exchange rate volatility and international trading strategy, Journal of International Money and Finance. 1991.

Johnston, J. DiNardo, J. métodos econométricos. São Paulo: Mc Graw Hill, 2001.

Jesus, L. O impacto da volatilidade cambial nas exportações brasileiras para o mercosul: uma análise em painel dinâmico. Dissertação de Mestrado, Universidade Federal do Paraná, Curitiba, PR, 2010.
Mendonça, T. G. Instituições e comércio bilateral de produtos agropecuários. $39^{\circ}$ Encontro Nacional de Economia, 2011.

Ministério das Relações Exteriores/Fundação Centro de Estudos de Comércio Exterior (FUNCEX/RJ). O Brasil e o NAFTA: Impacto sobre o Comércio e Investimentos. Brasília: ABRIGRAF, 1993.

Morettin, Pedro A. Econometria Financeira - Um Curso em Séries Temporais Financeiras. São Paulo: Blucher, 2008.

Peree, E.; A. Steinherr. Exchange rate uncertainty and foreign trade. European Economic Review 33, 1241-64, 1989.

Pindyck, Robert S.; Rubinfeld, D. L. Econometria. Rio de Janeiro: Elsevier, 2004.

Pinheiro, J. L. Mercado de Capitais: fundamentos e técnicas/Juliano Lima Pinheiro. $5^{\circ}$ ed. São Paulo: Atlas, 2009.

Ratti, B. Comércio Internacional e Câmbio. Brasil: Aduaneiras, 1997.

Toda, M. K. B. Impactos da Volatilidade da Taxa de Câmbio no Comércio Setorial do Mercosul e da Alca. Curitiba: UFPR. Relatório PIBIC/CNPq, 2012.

Trade Map. Disponível em: <http://www.trademap.org/ Index.aspx $>$.

Wei, S.J. Intra-national versus international trade: How stubborn are nations in global integration?. NBER Working Paper 5531, Cambridge, MA. NBER, 1996.

Willet, T.D. Exchange-Rate Volatility, International Trade. and Resource Allocation: A Perspective on Recent Research. Jounal of International,Money and Finance.Supplement, 5: SIOL-S112, March 1986.

Wooldridge, J. Introdução à econometria: uma abordagem moderna; tradução Rogério Cézar de Souza, José Antônio Ferreira. São Paulo: Pioneira Thomson Learning, 2006.

World Integration Trade Solution. Disponível em: $<$ http:// wits.worldbank.org/>. 